\title{
Improving muscle mass: response of muscle metabolism to exercise, nutrition and anabolic agents
}

\section{Kevin D. Tipton*1 and Arny A. Ferrando ${ }^{*}$}

*School of Sport and Exercise Sciences, University of Birmingham, Birmingham B15 2TT, U.K., and †Center for Translational Research in Aging and Longevity, Donald Reynolds Institute on Aging, Department of Geriatrics, University of Arkansas for Medical Sciences, $4301 \mathrm{~W}$. Markham St \#806, Little Rock, AR 72205, U.S.A.

\section{Abstract}

Muscle mass is critical for athletic performance and, perhaps more importantly for most, health and survival. The metabolic basis for a change in muscle mass is an increase in net muscle protein balance (termed NBAL). NBAL is the difference between MPS (muscle protein synthesis) and MPB (muscle protein breakdown). Thus an increase in MPS and/or a decrease in MPB are necessary for NBAL to increase, leading to accretion of muscle proteins. In particular, accretion of myofibrillar proteins is necessary. NBAL responds to exercise, feeding and other factors. In healthy, weight-stable adults, muscle mass remains constant because periods of positive balance following feeding are countered by periods of negative balance during fasting. A combination of resistance exercise and nutrition is a potent anabolic stimulus through stimulation of MPS from amino acids and attenuation of MPB by carbohydrates. Increased muscle mass results from the accumulation of small amounts of protein in response to each bout of exercise combined with nutrient intake. The magnitude of the ${ }^{1}$ To whom correspondence should be addressed (email k.d.tipton@bham.ac.uk). 
response may be influenced by factors other than just the amount of a nutrient ingested. Timing of ingestion, co-ingestion of nutrients and the type of protein may all influence protein accretion. Testosterone is a potent anabolic stimulus primarily through improvement in re-utilization of amino acids from MPB. There is a general lack of efficacy in studies assessing the potential for growth hormone, androstenedione and dehydroepiandrostenedione to increase muscle mass. Creatine supplementation is clearly an effective means to increase muscle mass, especially in combination with resistance exercise, however the mechanisms remain unclear. Results from acute metabolic studies provide useful information for estimation of the efficacy of anabolic agents.

\section{Introduction}

Muscle mass is critical for human health, physical activity and function, as well as for athletic performance [1]. Improvement of muscle mass is of great interest to many varied populations. Athletes and body builders desire to increase muscle mass and strength for competitive reasons. Others, such as the elderly, astronauts or those recovering from restricted activity or illness, need to increase muscle mass to increase functionality and quality of life. In these more stressed populations, muscle serves as a metabolic reserve to ensure glucose homoeostasis and to provide amino acid precursors for splanchnic, immunological and wound-healing requirements. Thus in circumstances where muscle mass has declined, a subsequent increase in muscle mass is necessary to regain life functions.

The metabolic basis for changes in muscle mass is net muscle protein balance (termed NBAL). Muscle proteins, in fact all body proteins, are constantly being synthesized and degraded. The balance between these two processes determines the amount of proteins in muscle. More specifically, changes in muscle mass are due to changes in the balance of the synthesis and breakdown of muscle myofibrillar proteins. Over any given time, the quantity of muscle protein is due to a change in NBAL. Accretion of muscle proteins occurs during periods of positive balance and muscle proteins are lost during periods of negative balance. Nutritional intake and exercise both have profound influences on the duration and magnitude of these periods of positive and negative NBAL. Additionally, changes in muscle mass are effected in great part by endocrine signals.

In this chapter we will examine the impact of exercise, nutrition and anabolic agents, including hormones and creatine, on muscle hypertrophy. We will focus primarily on the metabolic mechanisms responsible for muscle accretion and, whenever possible, base the discussion on studies in humans.

\section{Feeding and exercise}

Nutrition clearly has an enormous impact on changes in muscle mass. Recent research has helped elucidate changes in NBAL that result from nutritional 
manipulations [1a]. Daily changes in NBAL are a consequence of fasting and feeding. In the post-absorptive state, NBAL is negative, but feeding results in positive NBAL. Thus muscle protein is alternately deposited and released depending on the metabolic situation. The magnitude and duration of these periods of muscle protein deposition and release determine the amount of muscle mass (Figure 1). In healthy, weight-stable adults, the periods of positive and negative NBAL are approximately equal and muscle mass does not change over time. Manipulation of exercise and nutrition may change the magnitude and direction of the positive and negative periods of NBAL and thus change muscle mass. Increased amino acid availability stimulates MPS (muscle protein synthesis) and results in positive NBAL [2], i.e. accretion of muscle proteins. Other nutrients influence the response of NBAL to feeding. Insulin from carbohydrate ingestion has been demonstrated to increase MPS at rest provided amino acid availability is maintained [3]. There is no indication that lipid ingestion has an impact on NBAL at rest [4].

Resistance training results in increased muscle mass due to a combination of exercise and nutrient intake that reduces the basal, post-absorptive periods of negative NBAL and increases the periods of positive NBAL following feeding. The response of muscle protein metabolism to exercise and various nutrients is summarized in Table 1. Thus muscle hypertrophy occurs as a result of an accumulation of proteins in response to each individual exercise bout. MPS and MPB (muscle protein breakdown) may be increased during recovery following resistance exercise of sufficient intensity [5]. However, MPS is increased more than MPB, so NBAL is improved. In the absence of nutrient intake NBAL does not reach positive levels [5]. However, the improvement in NBAL following resistance exercise during post-absorptive periods reduces the negative NBAL, i.e. reduces the loss of amino acids during fasting periods. Provision of amino acids following exercise results in positive NBAL that is greater than that with amino acids at rest $[2,6,7]$. In other words, there is an interactive effect of resistance exercise and amino acids. Following resistance exercise, the response of MPS to hyperinsulinaemia is limited, probably due to amino acid availability. However, the response of MPB to resistance exercise is ameliorated [3]. Thus meals containing protein as a source of amino acids and carbohydrates to stimulate insulin release will increase MPS and decrease MPB to engender an even greater positive NBAL. Consequently, muscle hypertrophy stems from the accumulation of muscle protein as a result of the interaction of resistance exercise and nutrient intake, in that the increased periods of positive NBAL are combined with the ameliorated negative balance during post-absorptive periods following resistance exercise [8].

The possible metabolic explanations for the long-term increase in muscle mass are illustrated schematically in Figure 1. One explanation is that training induces chronic changes in NBAL. If so, then either the basal (resting, post-absorptive) balance (Figure 1A), or the response to each individual bout of exercise plus feeding (Figure 1B), would increase over time. Muscle mass 

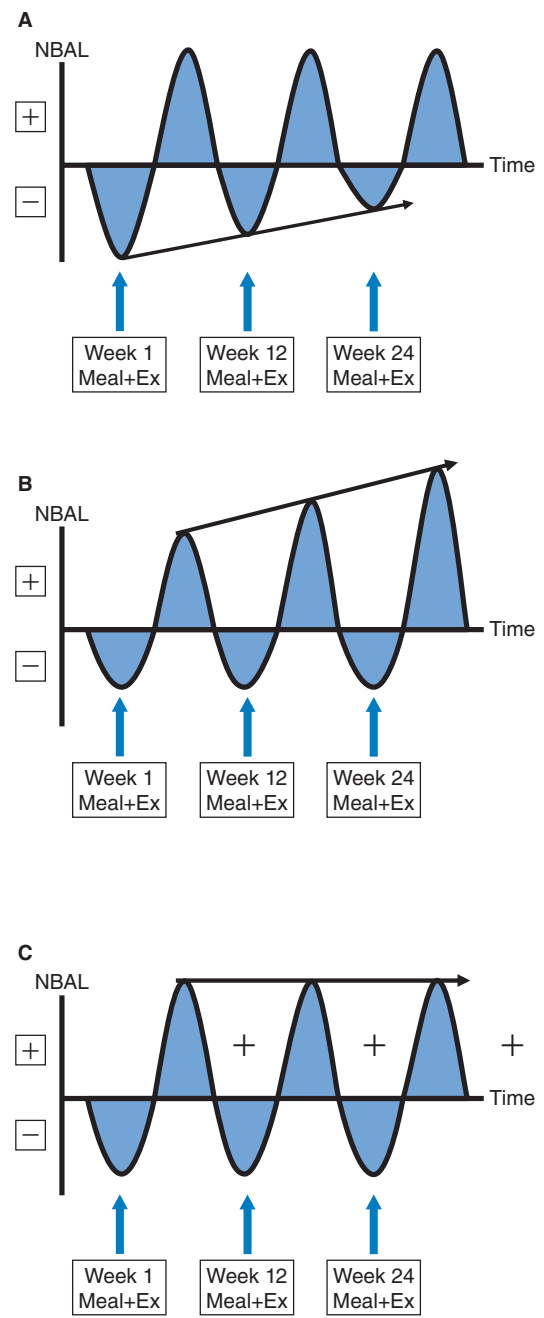

Figure I. Schematic depiction of possible metabolic mechanisms responsible for accretion of muscle protein with resistance training

(A) Muscle protein accretion due to an increase in basal, i.e. resting, post-absorptive NBAL. (B) Muscle protein accretion due to a chronic change over time in the response of NBAL to resistance exercise and nutrient (amino acid) intake. (C) Depiction of muscle protein gains due to the accumulation of muscle protein in response to each individual bout of resistance exercise plus nutrient (amino acid) intake. For illustration purposes, each depicts the theoretical daily response to resistance exercise plus ingestion of a meal at weeks I, 12 and 24 of training. In (A) the basal net balance changes, but the response to a meal plus exercise remains the same. In (B) the basal NBAL does not change, but the response to a meal plus exercise increases over time. In (C) there is no change in basal NBAL or in the response to a meal plus exercise, but instead muscle hypertrophy results from an accumulation of proteins due to the response to each individual exercise bout. Ex, exercise.

would then accrue progressively over time. Previously, we demonstrated that basal NBAL in trained weightlifters is no different from untrained volunteers [12]. In a more recent investigation from our laboratory using a longitudinal design, we demonstrated that 16 weeks of resistance training did not change 


\begin{tabular}{|c|c|c|c|}
\hline \multicolumn{4}{|c|}{$\begin{array}{l}\uparrow, \text { increase in rate; } \downarrow \text {, decrease in rate; } \leftrightarrow \text {, little to no change in rate; }+ \text {, NBAL } \\
\text { improves to positive levels from negative. }\end{array}$} \\
\hline & MPS & MPB & NBAL \\
\hline Protein or amino acids alone $[2,36]$ & $\uparrow$ & $\leftrightarrow$ or $\downarrow$ & $\uparrow$ to + \\
\hline Exercise alone $[5,37,38]$ & $\uparrow$ & $\uparrow$ & $\uparrow$ \\
\hline Amino acids plus exercise $[2,39,40]$ & $\uparrow \uparrow$ & $\leftrightarrow$ & $\uparrow \uparrow$ to + \\
\hline Carbohydrates (insulin) alone $[41,42]$ & $\uparrow$ & $\leftrightarrow$ & $\uparrow$ \\
\hline \multicolumn{4}{|l|}{ Carbohydrates (insulin) plus exercise } \\
\hline$[7,41,43]$ & $\leftrightarrow$ & $\downarrow$ & $\uparrow$ \\
\hline \multicolumn{4}{|l|}{ Carbohydrates (insulin) plus amino acids } \\
\hline following exercise $[7,10,44]$ & $\uparrow \uparrow$ & $\downarrow$ & $\uparrow \uparrow \uparrow$ to + \\
\hline
\end{tabular}

basal NBAL (K.D. Tipton, S.E. Wolf and R.R. Wolfe, unpublished work). Therefore it is unlikely that chronic changes in basal NBAL (Figure 1A) or in the response of NBAL to exercise and nutrition (Figure 1B) contribute to muscle hypertrophy.

An alternative explanation is that adaptations to training occur as a result of the summation of the response to each individual exercise bout (Figure 1C) [8]. This notion is supported by changes in molecular signalling pathways and gene expression in response to acute exercise [9]. Deposition of muscle protein following each bout of exercise is small and the accumulation of these small changes gradually increases muscle mass over a period of training with no detectable change in basal NBAL. This gradual accumulation of new proteins may explain why changes in muscle mass with training are generally not measurable until after several weeks of training [10], although it is worth noting that Seynnes et al. [10a] did recently demonstrate measurable increases in muscle mass following less than three weeks of resistance exercise training. This increase in muscle mass was much earlier than had previously been reported, thus supporting the notion that mass begins increasing early in a training period.

Muscle anabolism due to exercise and nutrient intake is influenced by various other factors, such as timing of ingestion, type of protein or amino acids ingested and co-ingestion of other nutrients. For example, milk protein ingestion following resistance exercise results in greater amino acid uptake by muscle than ingestion of soy protein [9]. Timing of ingestion may also influence the accretion of proteins. Amino acid uptake is greater when free amino acids and carbohydrates are ingested prior to rather than following resistance exercise [10]. However, the difference in amino acid uptake between ingestion of whole proteins prior to and following exercise does not seem to be as clear [11]. Thus the influence of timing of nutrient ingestion may be determined by the nature of the protein or amino acid that is ingested. Co-ingestion of other nutrients will also influence the response. Utilization of ingested amino acids is increased 
when carbohydrates are ingested with the amino acids $[6,7,10]$. Preliminary evidence suggests that utilization of amino acids from protein sources may also be increased when fat is ingested along with the protein [12]. This finding is interesting, but somewhat unexpected. At this point, a clear metabolic or molecular explanation for an increase in amino acid utilization from proteins co-ingested with lipids is lacking. Nevertheless, it seems that there is some influence of lipid co-ingested with protein on muscle anabolism following exercise [12]. These results illustrate that muscle hypertrophy due to resistance training is not as simple as merely ingesting a prescribed amount of protein in the diet. The interaction of several nutritional factors will influence the response and help determine the level and rate of hypertrophy during training. The mechanisms for these differences in anabolism due to ingestion of different types of protein remain to be elucidated. Certainly, molecular signalling events in response to amino acid ingestion, particularly leucine, resistance exercise $[14,15]$ and insulin or IGF-1 (insulin-like growth factor 1) [15] have been recently implicated in these responses. However, the complexity of these systems and the difficulty in determining the timing of the interactions of signalling and MPS in humans in vivo has left much to be determined.

\section{Anabolic agents}

The scientific evidence concerning the efficacy of anabolic agents has historically been at odds with the anecdotal evidence. This is particularly true in the discussion of testosterone. The results from earlier studies investigating the effects of testosterone were equivocal due to shortcomings in several design issues, such as standardization and control of dietary intake, control of testosterone formula, dosage and duration, adequate training standardization, and sensitivity of measurement techniques. However, within the last decade, the efficacy of testosterone has been demonstrated in several eloquent research studies. These studies indicate that testosterone administration leads to an increase in lean body mass, muscle size and muscle strength. Although testosterone, as with most other hormones, has been principally studied in the context of clinically deficient populations, studies conducted in the laboratory of Dr Shalendar Bhasin have delineated the efficacy and mechanisms of actions in a more traditional population. When administered in double-blind fashion to eugonadal men 19-40 years of age, a supraphysiological dose increased fat-free mass, muscle size and muscle strength [16]. These increases were further improved when combined with resistance training [16]. Subsequent multivariate analyses indicate that the anabolic response with testosterone is largely predicted by the dose [17]. Although a dose-response relationship has been demonstrated with testosterone, it is prudent to interpret these results cautiously. These were relatively short-term studies (10 weeks) and although no adverse side-effects were noted [16], the applicability of these findings to athletes must be interpreted with care, as athletes are notorious for their chronic use and abuse of testosterone to the point of clinical problems [18]. 
The action of testosterone on skeletal muscle indicates that several mechanisms are responsible for muscle hypertrophy and body composition changes. Testosterone induces muscle fibre hypertrophy through an increase in myoblast differentiation, an increase in satellite cell number, a proportionate increase in myonuclear number and changes in satellite cell ultrastructure [19]. Testosterone mediates body compositional changes by affecting the differentiation of mesenchymal-derived pluripotent stem cells. These cells, which are present in both muscle and fat tissue, are committed to the myogenic lineage and inhibited from the adipogenic lineage with testosterone usage [19]. This finding is consistent with the demonstrated reciprocal changes in fat and muscle mass with testosterone supplementation [16].

There is a widespread belief among athletes that testosterone supplementation enables a greater duration and intensity of training. However, existing data indicate that the primary benefit to the athlete is the increase in NBAL and muscle remodelling. In terms of muscle protein metabolism, administration of testosterone to young volunteers eliminates the loss of amino acids from muscle in the fasted state [20]. There is a substantial increase in MPS which is facilitated by an increased re-utilization of amino acids derived from MPB [20]. In other words, testosterone promotes MPS and turnover in part by increasing the efficiency of amino acid utilization in skeletal muscle [20]. An increase in protein turnover is inherent in the remodelling process in skeletal muscle. This effect in the fasted state enables greater muscle recovery by ameliorating the 'cost' of fasting, i.e. the loss of nitrogen and amino acids from skeletal muscle. This is a principal and chronic mechanism of action of testosterone on skeletal muscle protein metabolism, and one which is not induced by acute administration [21]. Thus in addition to its effects on muscle hypertrophy, enhanced muscle recovery is a beneficial aspect of testosterone supplementation. Testosterone does not enhance the effects of amino acid intake (derived by supplementation or high-protein diet), and, in fact, the amino acid effects on NBAL far surpass the anabolic effect of testosterone [21].

Unlike testosterone, the effects of GH (growth hormone) are not derived from thorough research in representative populations. Despite a commonly held belief that it is widely used in athletic endeavours, the clinical and research evidence for the effects of $\mathrm{GH}$ on skeletal muscle is underwhelming and may even be considered detrimental for athletes [22]. Certainly, administration of GH does not increase muscle protein synthesis [23], as does testosterone [20]. Muscle mass and strength increases with resistance training are no greater with $\mathrm{GH}$ administration than without GH [22]. It is probable that increases in body weight with GH administration are due to fluid retention and not an increase in muscle mass [22]. The general consensus is that there is a lack of evidence of GH effects on skeletal muscle. A recent review concludes that " $\mathrm{GH}$ administration alone or in combination with resistance exercise has little, if any, effect on muscle volume, strength, and fibre composition in non-GH-deficient healthy young individuals" [24]. It is worth noting, 
however, that this conclusion is analogous with the earlier consensus of the research community on the benefits of testosterone.

An increasingly common athletic practice is the combination of testosterone and GH. Although there is no evidence of increased performance efficacy when the two hormones are combined, there is clinical evidence suggesting a benefit in combining these therapies in older individuals. The combination of $\mathrm{GH}$ and testosterone has been demonstrated to increase fat-free mass [25], as well as muscle volume, strength and $\dot{V}_{\mathrm{O}_{\max }}[26]$ in elderly men. In general, examination of the modest findings supporting the effect of $\mathrm{GH}$ alone, along with the greater outcomes associated with the interaction of $\mathrm{GH}$ and testosterone, point to testosterone as the hormone primarily responsible for body composition and performance changes. The applicability of these findings to athletic performance is unknown since this research focuses primarily on hormone replacement or normalization in populations that are deficient. The response from hormonal augmentation in normal or athletic populations is likely to be different.

\section{Over-the-counter possibilities}

Owing in large part to recent exposure from marquee athletes (top class) the testosterone precursors DHEA (dehydroepiandrostenedione) and Andro (androstenedione) have received a great deal of attention as potential anabolic agents. The interest is further fuelled by the availability of these substances in over-the-counter preparations. DHEA and Andro are both produced by the gonads and adrenal glands and are precursors in the pathway of testosterone biosynthesis. As prohormones, or steroid precursors, both are touted as legitimate means of increasing 'natural' testosterone production in the body. Though these compounds have relatively weak anabolic activity by themselves, many studies have sought to discern whether their possible conversion into testosterone would increase blood testosterone and impact lean mass and strength gains. A recent review of this literature indicates unequivocally that Andro administration does not increase blood testosterone levels, or lead to an increase in strength or muscle mass [27]. One such study investigated the effects of 5 days of Andro on skeletal muscle metabolism [28]. Plasma testosterone levels were unchanged after Andro supplementation, and there was no effect on skeletal MPS or MPB, indicating the absence of an anabolic effect [28]. These results contrast with administration of testosterone that clearly increased MPS and NBAL in this short time period [20]. Furthermore, it seems clear that oral Andro results in a significant increase in blood oestrogen levels $[27,28]$. An increase in oestrogen is undesirable in males as it is associated with gynocomastia and other feminizing effects.

The studies examining DHEA are equally unimpressive. The recommended dosage is between 50 and $100 \mathrm{mg} / \mathrm{day}$, but dosages as high as $1600 \mathrm{mg} / \mathrm{day}$ have failed to show an increase in serum testosterone concentrations in men [29]. Further studies indicate that DHEA ingestion neither increases 
testosterone levels, nor impacts the adaptations associated with resistance training in young men [30]. Overall, DHEA is ineffective as an anabolic agent in both men and women, despite the increased conversion into testosterone in older women.

Of all the over-the-counter supplements, creatine has demonstrated a consistent ergogenic effect on exercise performance that may be mediated by increased muscle hypertrophy. Due to the large volume of research studies with creatine monohydrate, the reader is referred to a recent review by Volek and Rawson [31] which provides excellent summaries of the findings. The mechanism by which creatine supplementation increases muscle fibre hypertrophy during training remains unclear. Volek and Rawson [31] propose several potential mechanisms of action which explain the effects of creatine on muscle growth. However, direct evidence for increased MPS in humans is lacking [32,33]. Whatever the proposed mechanism(s) of action, the response to creatine supplementation is largely dependent on the degree of skeletal muscle creatine uptake [31]. The increase in muscle creatine content after supplementation is inversely related to the initial muscle creatine content. In other words, those with higher muscle creatine concentrations are less likely to further increase this concentration with creatine supplementation. There is also increasing evidence that an insulin response (generated by carbohydrate and/or protein ingestion) increases muscle accumulation of creatine [31]. For this reason, over-the-counter creatine supplements often contain carbohydrates to exploit this response. Overall, there is substantial research to indicate that creatine supplementation provides an ergogenic effect when muscle creatine levels are substantially elevated. The mechanism for this effect, however, remains to be elucidated.

\section{Methodology}

Much of the discussion above is based on studies that acutely measure changes in NBAL, MPS and MPB in response to exercise, nutrition or hormonal perturbations, or some combination thereof. Ideally, investigation of manipulations to improve muscle mass would involve long-term, longitudinal end-point studies with large numbers of subjects. However, the control of that type of investigation is all but impossible, the end-point measurements relatively imprecise, and the costs prohibitive. Therefore one potential problem with longitudinal end-point studies is that the results may not be representative of the actual physiological situation. Many of these studies fail to show a difference between treatment groups, but this lack of measurable difference does not necessarily indicate that no difference exists. A lack of measurable difference may simply mean that a real difference between groups was not detected because of lack of sufficient control, small number of subjects, imprecise measurement of end-points or the inability to carry out the study for sufficient time. As a result of these difficulties, the results from these studies are often quite equivocal. In recent years, many laboratories have investigated 
nutritional, exercise and hormonal interventions to improve muscle mass utilizing acute studies lasting only several hours. These investigations often make use of stable isotopic tracers, arteriovenous balance and/or muscle biopsies to examine the changes in muscle metabolism due to an intervention. Accordingly, the assumption is made that changes in metabolism observed during these short periods represent the potential for long-term changes in muscle mass if the particular intervention were maintained over weeks, months or years. Thus the validity of extrapolating results from acute studies to chronic changes/outcomes may be questioned.

Previously, we investigated the relationship between acute and $24 \mathrm{~h}$ changes in muscle protein metabolism. MPS and NBAL were compared in volunteers over a $24 \mathrm{~h}$ period under two conditions: (i) while resting; and (ii) during a $24 \mathrm{~h}$ period when they performed resistance exercise and ingested EAAs (essential amino acids) [34]. Results from a $3 \mathrm{~h}$ period after intervention were compared with those over the entire $24 \mathrm{~h}$ period. EAA ingestion and exercise increased $24 \mathrm{~h}$ MPS and improved NBAL over $24 \mathrm{~h}$. More importantly, the difference between rest and exercise was identical whether measured over $3 \mathrm{~h}$ or $24 \mathrm{~h}$ (Figure 2) [34]. These results support the contention that acute changes in NBAL represent those that occur over longer time periods.

If acute changes in NBAL are to be deemed representative of long-term changes, then the response of NBAL before and after an intervention, e.g.

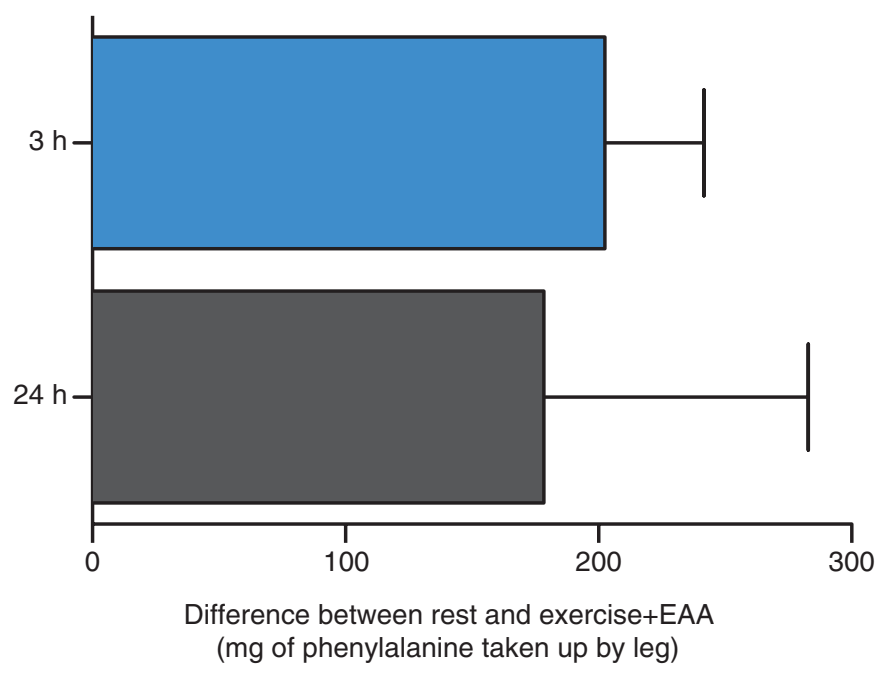

Figure 2. The difference in amino acid uptake between rest and resistance exercise plus EAA ingestion for a $3 \mathrm{~h}$ period from the beginning of exercise and for an entire $24 \mathrm{~h}$ period.

These results indicate the accretion of muscle protein over a $24 \mathrm{~h}$ period that includes resistance exercise and EAA is due primarily to the acute response. Adapted from Tipton, K.D., Borsheim, E., Wolf, S.E., Sanford, A.P and Wolfe, R.R. (2003) Acute response of net muscle protein balance reflects $24 \mathrm{~h}$ balance after exercise and amino acid ingestion. Am. J. Physiol. Endocrinol. Metab. 284, E76-E89 with permission. 
resistance-exercise training, must be constant. That is, if the response changes over a period of training (or dietary manipulation), such as in Figure 1(B), then the response measured acutely prior to the training could not be extrapolated to indicate potential changes in muscle mass that result from a period of training. In a recent study, the response of NBAL to resistance exercise and EAA ingestion was measured prior to and following 16 weeks of resistanceexercise training (K.D. Tipton, S.E. Wolf and R.R. Wolfe, unpublished work). Following training, the response of NBAL to resistance exercise and EAA was similar to that prior to training (illustrated schematically in Figure 1C). These findings indicate that results from a study in the untrained state could be extrapolated to estimate changes in muscle mass over time. Further support is provided from another previous study [35] in which NBAL was measured prior to and following 28 days of bed rest with and without EAA supplementation. EAA supplementation resulted in positive NBAL both prior to and following bed rest, but NBAL was reduced following bed rest. A comparison of estimates of muscle mass lost during bed rest from an extrapolation based on the initial acute NBAL measurement and an actual measurement using DEXA (dual energy X-ray absorptiometry) revealed very similar results [35]. These findings provide direct evidence that acute measurements may be useful for estimation of chronic changes in muscle mass.

Taken together, the results of these studies combined with the uncertainty of longitudinal measurements demonstrate the utility of acute studies in determining the impact of various nutritional, exercise or endocrine influences on muscle mass. Acute studies may be used to determine the potential for interventional changes, while providing useful mechanistic information. In the absence of the requisite logistical and economical support necessary to conduct longitudinal studies, assessment of the acute response to an endocrine, exercise and/or nutritional intervention may be used to elucidate mechanistic changes in muscle protein metabolism, as well as providing information on the potential for long-term changes.

\section{Summary}

- Maintenance of muscle mass is critical for bealth, function and atbletic performance.

- The metabolic basis for changes in muscle mass is the balance between the rates of protein synthesis and breakdown.

- Increased muscle mass during training results from the accumulation of protein in response to each individual bout of exercise.

- Resistance exercise alone improves muscle protein balance, but in the absence of an amino acid source, balance does not reach positive levels.

- Exogenous amino acids stimulate MPS resulting in positive muscle protein balance.

- Insulin stimulates MPS at rest, but following exercise the increase is minimal without additional amino acids. 
- The largest response of net muscle protein balance is due to ingestion of a combination of amino acids and carbobydrates.

- Timing of nutrient ingestion in relation to exercise and other nutrients plays a key role in the response of net muscle protein balance.

- Testosterone increases the re-utilization of amino acids for protein synthesis resulting in increased muscle mass.

- There is little evidence for efficacy of growth hormone, androstenedione or DHEA for stimulation of muscle protein synthesis.

- Acute metabolic studies provide important information for predicting long-term changes in muscle mass.

\section{References}

I. Wolfe, R.R. (2006) The underappreciated role of muscle in health and disease. Am. J. Clin. Nutr. 84, 475-482

Ia. Tipton, K.D. \& Wolfe, R.R. (2004) Protein and amino acids for athletes. J. Sports Sci. 22, 65-79

2. Biolo, G., Tipton, K.D., Klein, S. \& Wolfe, R.R. (1997) An abundant supply of amino acids enhances the metabolic effect of exercise on muscle protein. Am. J. Physiol. 273, EI22-EI29

3. Biolo, G., Williams, B.D., Fleming, R.Y. \& Wolfe, R.R. (1999) Insulin action on muscle protein kinetics and amino acid transport during recovery after resistance exercise. Diabetes 48, 949957

4. Svanberg, E., Moller-Loswick, A.C., Matthews, D.E., Korner, U., Andersson, M. \& Lundholm, K. (1999) The role of glucose, long-chain triglycerides and amino acids for promotion of amino acid balance across peripheral tissues in man. Clin. Physiol. 19, 31 I-320

5. Biolo, G., Maggi, S.P., Williams, B.D., Tipton, K.D. \& Wolfe, R.R. (I995) Increased rates of muscle protein turnover and amino acid transport after resistance exercise in humans. Am. J. Physiol. 268, E5I4-E520

6. Borsheim, E., Tipton, K.D., Wolf, S.E. \& Wolfe, R.R. (2002) Essential amino acids and muscle protein recovery from resistance exercise. Am. J. Physiol. Endocrinol. Metab. 283, E648-E657

7. Miller, S.L., Tipton, K.D., Chinkes, D.L., Wolf, S.E. \& Wolfe, R.R. (2003) Independent and combined effects of amino acids and glucose after resistance exercise. Med. Sci. Sports Exercise $\mathbf{3 5}$, 449-455

8. Phillips, S.M. (2004) Protein requirements and supplementation in strength sports. Nutrition 20, 689-695

9. Phillips, S.M., Hartman, J.W. \& Wilkinson, S.B. (2005) Dietary protein to support anabolism with resistance exercise in young men. J. Am. Coll. Nutr. 24, I34S-139S

10. Tipton, K.D., Rasmussen, B.B., Miller, S.L., Wolf, S.E., Owens-Stovall, S.K., Petrini, B.E. \& Wolfe, R.R. (200I) Timing of amino acid-carbohydrate ingestion alters anabolic response of muscle to resistance exercise. Am. J. Physiol. Endocrinol. Metab. 28I, EI97-E206

10a. Seynnes, O.R., de Boer, M. \& Narici, M.V. (2007) Early skeletal muscle hypertrophy and architectural changes in response to high-intensity training. J. Appl. Physiol. 102, 368-373

II. Tipton, K.D., Elliott, T.A., Cree, M.G., Aarsland, A.A., Sanford, A.P. \& Wolfe, R.R. (2007) Stimulation of net muscle protein synthesis by whey protein ingestion before and after exercise. Am. J. Physiol. Endocrinol. Metab. 292, E7I-E76

12. Elliott, T.A., Cree, M.G., Sanford, A.P., Wolfe, R.R. \& Tipton, K.D. (2006) Milk ingestion stimulates net muscle protein synthesis following resistance exercise. Med. Sci. Sports Exercise 38, 667-674

13. Reference deleted

14. Karlsson, H.K., Nilsson, P.A., Nilsson, J., Chibalin, A.V., Zierath, J.R. \& Blomstrand, E. (2004) Branched-chain amino acids increase p70S6k phosphorylation in human skeletal muscle after resistance exercise. Am. J. Physiol. Endocrinol. Metab. 287, EI-E7 
I5. Kimball, S.R., Farrell, P.A. \& Jefferson, L.S. (2002) Role of insulin in translational control of protein synthesis in skeletal muscle by amino acids or exercise. J. Appl. Physiol. 93, II68-1 I80

16. Bhasin, S., Storer, T.W., Berman, N., Callegari, C., Clevenger, B., Phillips, J., Bunnell, T.J., Tricker, R., Shirazi, A. \& Casaburi, R. (1996) The effects of supraphysiological doses of testosterone on muscle size and strength in normal men. N. Engl. J. Med. 335, I-7

17. Woodhouse, L.J., Reisz-Porszasz, S., Javanbakht, M., Storer, T.W., Lee, M., Zerounian, H. \& Bhasin, S. (2003) Development of models to predict anabolic response to testosterone administration in healthy young men. Am. J. Physiol. Endocrinol. Metab. 284, 1009-1017

18. Rogol, A.D. \& Yesalis, 3rd, C.E. (1992) Clinical review 3I: anabolic-androgenic steroids and athletes: what are the issues? J. Clin. Endocrinol. Metab. 74, 465-469

19. Herbst, K.L. \& Bhasin, S. (2004) Testosterone action on skeletal muscle. Curr. Opin. Clin. Nutr. Metab. Care 7, 27I-277

20. Ferrando, A.A., Tipton, K.D., Doyle, D., Phillips, S.M., Cortiella, J. \& Wolfe, R.R. (1998) Testosterone injection stimulates net protein synthesis but not tissue amino acid transport. Am. J. Physiol. 275, E864-E87I

21. Wolfe, R.R., Ferrando, A.A. \& Urban, R.J. (2000) Testosterone and muscle protein metabolism. Mayo Clin. Proc. 75, S55-S60

22. Yarasheski, K.E. (1994) Growth hormone effects on metabolism, body composition, muscle mass, and strength. Exercise Sport Sci. Rev. 22, 285-312

23. Yarasheski, K.E., Zachweija, J.J., Angelopoulos, T.J. \& Bier, D.M. (1993) Short-term growth hormone treatment does not increase muscle protein synthesis in experienced weight lifters. J. Appl. Physiol. 74, 3073-3076

24. Weber, M.M. (2002) Effects of growth hormone on skeletal muscle. Horm. Res. 58 (Suppl. 3), 43-48

25. Brill, K.T., Weltman, A.L., Gentili, A., Patrie, J.T., Fryburg, D.A., Hanks, J.B., Urban, R.J. \& Veldhuis, J.D. (2002) Single and combined effects of growth hormone and testosterone administration on measures of body composition, physical performance, mood, sexual function, bone turnover, and muscle gene expression in healthy older men. J. Clin. Endocrinol. Metab. 87, 5649-5657

26. Giannoulis, M.G., Sonksen, P.H., Umpleby, M., Breen, L., Pentecost, C., Whyte, M., McMillan, C.V., Bradley, C. \& Martin, F.C. (2006) The effects of growth hormone and/or testosterone in healthy elderly men: a randomized controlled trial. J. Clin. Endocrinol. Metab. 91, 477-484

27. Bahrke, M.S. and Yesalis, C.E. (2004) Abuse of anabolic androgenic steroids and related substances in sport and exercise. Curr. Opin. Pharmacol. 4, 6I4-620

28. Rasmussen, B.B., Volpi, E., Gore, D.C. \& Wolfe, R.R. (2000) Androstenedione does not stimulate muscle protein anabolism in young healthy men. J. Clin. Endocrinol. Metab. 85, 55-59

29. Nestler, J.E., Barlascini, C.O., Clore, J.N. \& Blackard, W.G. (1988) Dehydroepiandrosterone reduces serum low density lipoprotein levels and body fat but does not alter insulin sensitivity in normal men. J. Clin. Endocrinol. Metab. 66, 57-6I

30. Brown, G.A., Vukovich, M.D., Sharp, R.L., Reifenrath, T.A., Parsons, K.A. \& King, D.S. (1999) Effect of oral DHEA on serum testosterone and adaptations to resistance training in young men. J. Appl. Physiol. 87, 2274-2283

3I. Volek, J.S. \& Rawson, E.S. (2004) Scientific basis and practical aspects of creatine supplementation for athletes. Nutrition 20, 609-6/4

32. Louis, M., Poortmans, J.R., Francaux, M., Berre, J., Boisseau, N., Brassine, E., Cuthbertson, D.J., Smith, K., Babraj, J.A., Waddell, T. \& Rennie, M.J. (2003) No effect of creatine supplementation on human myofibrillar and sarcoplasmic protein synthesis after resistance exercise. Am. J. Physiol. Endocrinol. Metab. 285, El089-EI094

33. Parise, G., Mihic, S., MacLennan, D., Yarasheski, K.E. \& Tarnopolsky, M.A. (200I) Effects of acute creatine monohydrate supplementation on leucine kinetics and mixed-muscle protein synthesis. J. Appl. Physiol. 91, 104I-1047

34. Tipton, K.D., Borsheim, E., Wolf, S.E., Sanford, A.P. \& Wolfe, R.R. (2003) Acute response of net muscle protein balance reflects $24-\mathrm{h}$ balance after exercise and amino acid ingestion. Am. J. Physiol. Endocrinol. Metab. 284, E76-E89 
35. Paddon-Jones, D., Sheffield-Moore, M., Urban, R.J., Sanford, A.P., Aarsland, A., Wolfe, R.R. \& Ferrando, A.A. (2004) Essential amino acid and carbohydrate supplementation ameliorates muscle protein loss in humans during 28 days bedrest. J. Clin. Endocrinol. Metab. 89, 435I-4358

36. Tipton, K.D., Gurkin, B.E., Matin, S. \& Wolfe, R.R. (1999) Nonessential amino acids are not necessary to stimulate net muscle protein synthesis in healthy volunteers. J. Nutr. Biochem. 10, 89-95

37. Phillips, S.M., Tipton, K.D., Aarsland, A., Wolf, S.E. \& Wolfe, R.R. (1997) Mixed muscle protein synthesis and breakdown following resistance exercise in humans. Am. J. Physiol. 273, E99-EI07

38. Phillips, S.M., Tipton, K.D., Ferrando, A.A. \& Wolfe, R.R. (1999) Resistance training reduces the acute exercise-induced increase in muscle protein turnover. Am. J. Physiol. 276, EII8-EI 24

39. Tipton, K.D., Ferrando, A.A., Phillips, S.M., Doyle, Jr, D. and Wolfe, R.R. (1999) Postexercise net protein synthesis in human muscle from orally administered amino acids. Am. J. Physiol. 276, E628-E634

40. Tipton, K.D., Elliott, T.A., Cree, M.G., Wolf, S.E., Sanford, A.P. \& Wolfe, R.R. (2004) Ingestion of casein and whey proteins result in muscle anabolism after resistance exercise. Med. Sci. Sports Exercise 36, 2073-208I

4I. Biolo, G., Declan Fleming, R.Y. \& Wolfe, R.R. (1995) Physiologic hyperinsulinemia stimulates protein synthesis and enhances transport of selected amino acids in human skeletal muscle. J. Clin. Invest. 95, 8II-819

42. Wolfe, R.R. (2000) Effects of insulin on muscle tissue. Curr. Opin. Clin. Nutr. Metab. Care 3, 67-7I

43. Borsheim, E., Cree, M.G., Tipton, K.D., Elliott, T.A., Aarsland, A. \& Wolfe, R.R. (2004) Effect of carbohydrate intake on net muscle protein synthesis during recovery from resistance exercise. J. Appl. Physiol. 96, 674-678

44. Borsheim, E., Aarsland, A. \& Wolfe, R.R. (2004) Effect of an amino acid, protein, and carbohydrate mixture on net muscle protein balance after resistance exercise. Int. J. Sport Nutr. Exercise Metab. I4, 255-27I 